\title{
Computer Applications in the Distribution of Incompatibilities in the Works of ERP
}

\author{
Khaldoun Besoul \\ electrical engineering department / north jeddeh
}

\begin{abstract}
In this paper steady progress in a competitive market environment, the advanced of the enterprise around the world seek to reduce the cost of execution of processes and their management, and at the same time trying to improve the quality of their processes. [1] In the recent widespread use of the approach of "best practices» (Best Practices). This became possible, because were successfully automated many aspects of the enterprise. Managed to accumulate enough knowledge in each specific areas and to turn that knowledge into the programmatic products.

Applying at "best practices" of the enterprise uses the knowledge gained by other E-businesses. For most processes, this leads to better quality and in a decrease in costs. However, the market environment is a competitive. If all market participants will work exactly the same way, the prize will be determined by random selection consumer.

Thus, on the one hand, use of the "best practices" leads to lower, on the other hand - a weakening of competitive advantage. A paradox: the use of "best practices" - well, not using - too well. Output: enterprises will be more effective if we used a "best practice" in the processes that are not important in terms of competitive advantage
\end{abstract}

How can control the processes that ensure the competitive advantage of the company (called future practice» - Next Practices). They are governed by their discretion. Management processes such leadership company pays great attention was. For the management of business processes (Business Process Management) methodology developed by the same name, aimed at improving the efficiency of processes, including the methods, approaches, and software products for managing and continuously improving the performance of the enterprise. [2]

\section{1 - INTRUDACTION}

Modern integrated informational system should provide a single re-solution, which automates the process control at all stages of their life cycle. This solution includes the following technologies. - Modeling - building a model of the process model based on surveys of business models and prototypes. Model of the prototype, developed in relation to the conditions of the real or constructed theoretically, document process know-how, which is used for simulation [7]. Unraveling project-individualization process types as and when the conditions of its launch. It also determines the model process, assigned specific parameters of individual tasks and allocate resources to those of the problem. Each project is characterized by four main parameters: time, cost, quality and prestige. Kudos depends on the first three variables and objectives. Quality is controlled by the customer drawings and specifications. In such a way, only time and cost are Xia variables to be monitored. [8] In the definition of the process indicates which tasks should be performed. Is given by the procedure for their implementation. But in the definition of the process is silent on who the long-wives to perform these tasks. [3] Hence the importance of the following technologies - management workflow

- Workflow - realization of the process of automated workflow management system, using the possibility of an automated issuing assignments implementing, integration of input and output data and automated ion open applications. In current operations (Workflow) - is a process of working transitions which are under control of the application - system management workflow (Workflow Management System WfMS). The basis for modeling workflows are properly structured-centered process model $[4,5]$.

\section{3 - APRROACH}

\section{The distribution of resources}

By combining the above technologies are able to solve one of the most important tasks in process management - resource allocation. We consider two formulations of the problem of optimal allocation of resources to tasks, depending on the optimality criterion of the (cost and time). Suppose there is an acyclic I-graph (roadmap), whose vertices are the events and arcs - instances of tasks. Each tasks has its own complexity of execution. Need to find a distribution of the resource instance Run, Sun cost for copies of tasks, in which:

1) the cost of performing the process $S$ shall be taken by the minimum value;

2) while the process is minimal. 


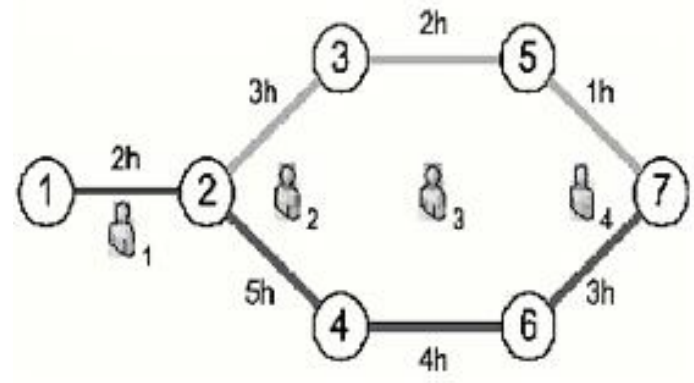

Fig. 1. Conditional sample process

Provided that the amount of resource j-type allocated to copy of the problem $(\mathrm{NRj}, \mathrm{i})$, not negative, and that the performance resource instance the task m-type $(\mathrm{Pn}, \mathrm{m})$ is positive. Consider a hypothetical example for illustrative ion features of the problem of resource allocation (Figure 1).

Here, the operation - it arcs connecting the nodes. Close to the time indicated by the arcs of operations. Various operations are timepersonal resources. In the process, there are two alternative routes 1-2-3-5-7 and 1-2-4-6-7 (nodes 2 and 7 are asynchronous $\mathrm{OR}$ ). Suppose that we need to process two parts, one on the first route, the second in the second. Arises the question of what part to start processing the first. Otherwise, the question can be formulated as follows: how to allocate resources for operations?

In this case, there are two ways. They are shown in Fig. 2.

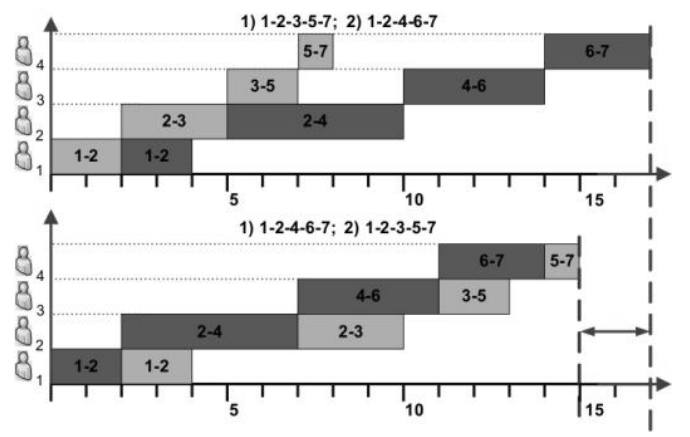

Fig. 2. Two variants of the allocation of resources

It can be seen that different allocation of resources leads to different run-time process. Thus, even at This simple example shows the importance of the optimal allocation of resources

\section{4 . method of resource allocation}

Dignity of heuristics - convenient implement them on a computer, even when solving problems of cumbersome, and a quick time. Disadvantages of heuristics are to estimate the closeness of the obtained optimal. In addition, for each preference functions there are problems for which the use of this function leads to poor results. [6]

This paper investigated features of the heuristic approach to the problem of distributed heterogeneous resources. An algorithm based on the dilation of priority rules. The algorithm is illustrated by the simple example with two parameters: the cost of the operation and the operation. Examples of them include a combination rule:

$\mathrm{a}$
$\mathrm{C} \max$ $\begin{array}{r}a \\ t_{\text {max }}\end{array}$

where ac - weighting for costs [0 .. 1]; at - weighting for time $[0 . .1]$; ci - cost for the th operation; ti - time to make good of the operation; cmax - maximum value

costs; tmax - the maximum time you-filled Show the work rules for a fragment of the process (see Fig. 1). Let the cost of the operation 50 at $01.02,2-3-300 \mathrm{y}$. e, 2-4 $100 \mathrm{y}$. is "satisfied, cornfields" operation 1-2, we are in a node 2. In this case, subject to implementation steps 2-3 and 2-4.

\begin{tabular}{|c|c|}
\hline$a_{c}=1 ; a_{t}=1$ & $a_{c}=0 ; a_{t}=1$ \\
\hline Rulefor $O p_{2-3}=\frac{1}{3} \quad 1+1=\frac{4}{3}$ & Rulefor $O p_{2-3}=\frac{1}{3} \quad 0+1=1$ \\
\hline Rulefor $O p_{2-4}=1+\frac{3}{5} 1=\frac{8}{5}$ & Rulefor $O p_{2-4}=0+\frac{3}{5} 1=\frac{3}{5}$ \\
\hline$\frac{4}{3}<\frac{8}{5}$ & $1>\frac{3}{5}$ \\
\hline $\begin{array}{c}\text { RuleforOp } 2-3< \\
\text { RuleforOp } 2-4\end{array}$ & $\begin{array}{c}\text { RuleforOp2-3 }> \\
\text { RuleforOp } p_{2-4}\end{array}$ \\
\hline Next steps 2-4 & Next steps 2-3 \\
\hline
\end{tabular}

Thus, by varying the weighting coefficients, we can obtain different solutions. Comparing these solutions, together, we can analyze the effects of different heuristics to solve the problem.

\section{5 .Research resource allocation heuristic method}

The initial data considered of copies of the conditional process. Conducted investigation of the influence of the 
weight coefficients of combined-centered priority in the results obtained. We used the following combinations rules:

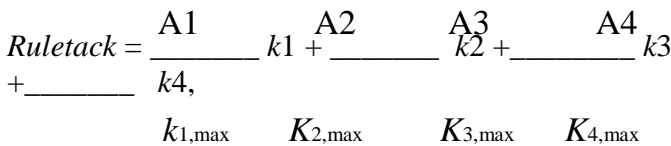

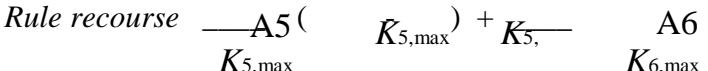

where ai - weighting factor [0 .. 1]; ki - value of the index for the object (task or of the resource); ki, max - the maximum value of the index for the available for tasks or free resources. Indicators:

1 - the complexity of the problem you-filled,

2 - number of resources chinch appointed to the task,

3 - the average cost for a task,

4 - full reserve tasks,

5 - the cost of the resource,

6 - the performance of the resource.

Investigated both objective functions: minimization of costs and minimize time. Here are the results for the minimization of the time. First crawl space options of varying weights. A study of the revealed that the variation coefficients of the priority rules significantly affect the value of the objective function. For example: in the worst case execution of a process instance would take $227 \mathrm{y}$. is Using

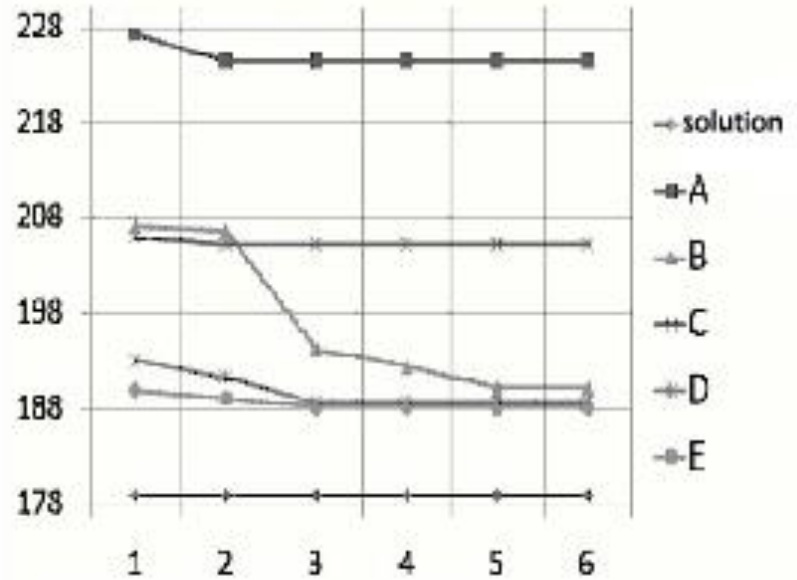

Fig. 3. The average value of (reduced time)

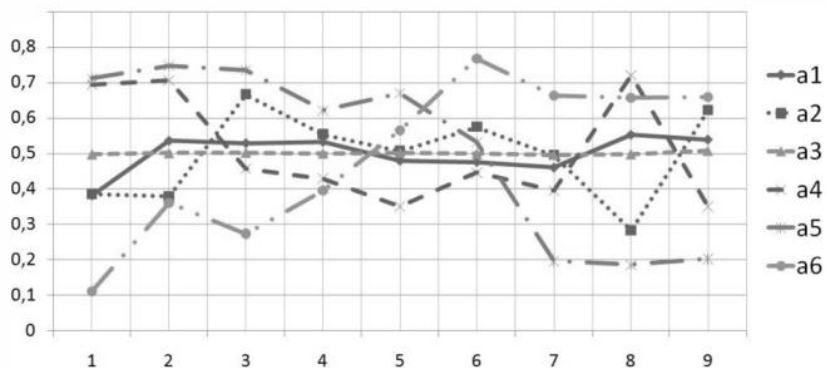

Fig. 4. Local search in five random points

developed program to obtain a solution for a 178. is, therefore, a difference the composition of about $25 \%$.

Further research was conducted effectiveness of the selection of priority rules. Space options split into nine sub-areas in each sub region calculated average weights (Fig.3 ) The diagrams show that less PWM values of the objective function of time corresponds to the lower value of weight coefficient for the fifth rule and more important factor for the sixth. The value of weight for the third rule is changed slightly. One can assume that the data in priority rule has no effect on the objective function and its use is inefficient Were also selected five points in the space of options and made the run local optimization algorithm (Figure 4)..

Seen from the figure, that in one of the five selected points not reached the lowest value of time. Moreover, noticeably that local optimum slightly improves the original value. This is explained by the fact that space options in many areas is quite extensive (the area with the same objective function value). This suggests that the local optimization algorithm needs improvement.

\section{RESULTS}

this study showed the promise of the approach to analyze the impact of various priority rules on the distribution of resources. The analysis for this particular problem can be a combination of rules-GET THERE, is the most appropriate solution. Like any algorithm, an algorithm developed by the authors, has a drawback: the complexity of the evaluation of the solution close to the optimum. Is obtained using the algorithm solutions, Vero yarn may be improved by constructing hyper-bring algorithm based on the developed and genetic algorithms. This topic is of interest for future research.

\section{REFERENCES}

[1] Scheer, A.W. Business Process Automation. ARIS in Practice [Text] / A.W. Scheer, F. Abolhassan, W. There [et al.]. -Berlin, 2004.

[2] Snabe, J.H. Business Process Management - Tae SAPRoadmap [Text] / JH Snabe, A. Rosenberg, C. Moliere [et al.]. -Boston, 2009.

[3] Bakry, A. H. and Bakry, S. H. (2005). "Enterprise resource planning - a review and a STOPE view," International Journal of Network Management 15.pp.363-370 
[4] Glass, R. L. (1998). "Enterprise Resource Planning Breakthrough or Term Problem," The Database for Advances in Information Systems, 29:2. Goodman, E. (2004). "Security Evaluation and Management for the SAP R/3 Environment," GSEC Certification Practical
[5] Shehab, E. M., Sharp, M. W., Supramaniam, L., and Spedding, T. A. (2004). "Enterprise resource planning an integrative review," Business Process Management Journal, 10:4,pp.359-386

[6] Sprott, D. (2000). "Componentizing the Enterprise Application Packages,"Communications of theACM,43:4. 